\title{
The effects of preconditioning with IFN- $\gamma$, IL-4, and IL-10 on costimulatory ligand expressions of mesenchymal stem cells
}

\author{
(ㄱ) Alper Tunga Ozdemir'1, Mustafa Oztatlici², (i) Rabia Bilge Ozgul Ozdemir ${ }^{3}$, (i) Busra Cakir ${ }^{4}$, \\ (1) Kemal Ozbilgin'2, (1) Ertan Dariverenli ${ }^{5}$, (1) Cengiz Kirmaz \\ 'Department of Medical Biochemistry, Merkezefendi State Hospital, Manisa, Turkey \\ ${ }^{2}$ Department of Histology and Embryology, Manisa Celal Bayar University Faculty of Medicine, Manisa, Turkey \\ ${ }^{3}$ Department of Allergy and Clinical Immunology, Manisa City Hospital, Manisa, Turkey \\ ${ }^{4}$ Department of Bioengineering, Ege University Faculty of Engineering, Izmir, Turkey \\ ${ }^{5}$ Department of Pharmacology, Manisa Celal Bayar University Faculty of Medicine, Manisa, Turkey \\ ${ }^{6}$ Department of Allergy and Clinical Immunology, Manisa Celal Bayar University Faculty of Medicine, Manisa, Turkey
}

\begin{abstract}
Objectives: Mesenchymal stem cells (MSCs) are strong immunomodulatory cells, and co-stimulation may play an important role in increasing the effects of MSCs on adaptive immune cells. Preconditioning may add to the effectiveness of MSCs. The aim of this study was to investigate alterations in the costimulatory ligand expressions of MSCs preconditioned with inflammatory cytokines.

Methods: MSCs were preconditioned with interferon gamma (IFN- $\gamma$ ), interleukin (IL) 4 (IL-4), and IL-10, and changes in CD80, CD86, CD137L, CD252, CD274, CD275, and human leukocyte antigen (HLA) class I and II expressions were analyzed using flow cytometry and quantitative polymerase chain reaction methods. Human acute monocytic leukemia cell line (THP-1) macrophages preconditioned under the same conditions served as a control for comparison.

Results: The frequencies of CD80 ( $p=0.0003), C D 86(p<0.0001), C D 137 L(p<0.0001), C D 252(p=0.0003), C D 274$ $(p=0.0077), C D 275(p<0.0001)$, and HLA-II $(p<0.0001)$-positive MSCs was significantly lower than that of the THP-1 macrophages with either method, but there was no significant difference in the HLA-I $(p=0.1506)$ cells. Comparison of the expression of the costimulatory ligands revealed that the expression of MSCs was significantly lower than that of THP-1 cells, and was not affected by cytokine stimuli.

Conclusion: The study data indicated that although MSCs are strong immunomodulatory cells, the costimulatory ligand expression required for an effective antigen presentation was extremely low compared with that of professional antigen presenting cells. In addition, preconditioning with IFN- $\gamma$, IL-4, and IL-10 failed to increase the expression of important costimulatory ligands, such as CD80 and CD86, in MSCs. The stability of costimulatory ligand expression suggests that MSCs may be an effective source for HLA-I-mediated peripheral tolerance.

Keywords: Costimulatory ligands, interferon gamma, interleukin 4, interleukin 10, mesenchymal stem cells, preconditioning
\end{abstract}

$\mathrm{M}^{\mathrm{s}}$ esenchymal stem cells (MSCs) are powerful immunomodulatory cells. Molecules such as prostaglandin E2 (PGE2), indoleamine-pyrrole 2,3-dioxygenase (IDO), transforming growth factor-beta (TGF- $\beta$ ), interleukin (IL) 10, and hepatocyte growth factor (HGF) play an important role in the formation of these effects [1-3]. Molecules on the cell surface of MSCs are another mechanism that suppresses immune cells through cell contact. Strong immunosuppressive molecules, such as programmed death-ligand 1 (PD-L1/CD274), human leukocyte antigen (HLA) G, and B7-Homolog 3 (CD276) are

Address for correspondence: Alper Tunga Ozdemir, MD. Department of Medical Biochemistry, Merkezefendi State Hospital, Manisa, Turkey Phone: +90 5052973269 E-mail: alpertungaozdemir@outlook.com ORCID: 0000-0002-7708-077X

Submitted Date: January 28, 2021 Accepted Date: March 24, 2021 Available Online Date: May 03,2021

${ }^{\circ}$ Copyright 2021 by International Journal of Medical Biochemistry - Available online at www.internationalbiochemistry.com

OPEN ACCESS This work is licensed under a Creative Commons Attribution-NonCommercial 4.0 International License. 
highly expressed by MSCs [4-6]. Therefore, they are used experimentally in the treatment of many autoimmune diseases, such as systemic lupus erythematosus, rheumatoid arthritis, multiple sclerosis, and Crohn's disease. However, most studies have reported that the clinical effects of MSCs are variable and disappear in 3 to 9 months [7]. Several approaches have been used to improve the effectiveness of MSCs. The most common is preconditioning with a specific stimulus, such as hypoxia, drugs, or biological factors $[8,9]$. Interferon gamma (IFN- $\gamma$ ) is the cytokine most studied, and IFN- $\gamma$ priming has been shown to upregulate the MSC expression of several immunosuppressive molecules, including IDO, cyclooxygenase 2 (COX-2), TGF- $\beta$, and HGF [9]. IFN- $\gamma$ is also effective on the surface molecules of MSCs. It has been reported that immune regulatory surface molecule expressions, such as PD-L1, HLA-G, vascular cell adhesion molecule 1 (VCAM-1), intercellular adhesion molecule 1 (ICAM-1), were increased with IFN- $\gamma$ stimulation $[6,10,11]$. In addition to IFN- $\gamma$, tumor necrosis factor alpha (TNF-a), IL-6, and IL-17, may be used. Although not as much as IFN- $\gamma$, these cytokines have also been shown to cause an increase in IDO, PGE2, IL-10, and TGF- $\beta$ expression [12-14].

T lymphocytes are responsible for the formation of the adaptive immune response, and require 3 different signals for activation. Signal 1 is provided by the T-cell receptor upon recognition of an antigen on MHC molecules. Signal 2 is generated by costimulatory molecules, such as CD80/CD86 (B7.1/B7.2), on antigen-presenting cells. The cytokines in the environment enable the third signal. If Signal 1 is not supported by Signal 2, anergy or tolerance against the presented antigen occurs [15]. MSCs are known to not express costimulatory molecules, and therefore induce anergy in T cells [16]. It has been reported that MSCs do not express MHC-II, but stimuli such as IFN- $\gamma$ can increase expression without affecting the expression of the costimulatory molecules CD80/CD86 [17]. CD80 and CD86 co-stimulation plays a critical role in shaping the activity of T cells. However, in addition to naive $T$ cells, various costimulatory molecules shape the activation of effector and memory T cells. CD137L (4-1BBL), CD252 (OX40L), CD274 (PD-L1), CD275 (inducible costimulator ligand [ICOSL]) have been studied extensively in the literature [18]. It is well known that the cytokines IFN- $\gamma$, IL-4, and IL-10 have direct effects on antigen presentation and shaping the $T$ cell phenotype, co-stimulation in dendritic cells (DCs), and macrophage activation [19]. However, the current literature does not reveal how MSCs express alternative costimulatory molecules or how these expressions might change with cytokine stimulation. The objective of this study was to investigate alterations in the costimulatory molecule expression of MSCs preconditioned with inflammatory cytokines. Human adipose tissue (AD) MSCs and THP-1 macrophages were stimulated with IFN- $\gamma$, IL-4, and IL-10 cytokines, and the effects of these stimuli on the expression of HLA, CD80, CD86, CD137L, CD252, CD274, and CD275 molecules was evaluated. The costimulatory molecule expression of MSCs were compared in different inflammatory environments to THP-1 macrophages, a professional antigen-presenting cell (APC) model.

\section{Materials and Methods}

\section{Cell culture}

AD-MSCs (PCS-500-011; American Type Culture Collection, Manassas, VA, USA) and THP-1 cells (TIB-202; American Type Culture Collection, Manassas, VA, USA) were procured. The ADMSCs were cultured using Dulbecco's Modified Eagles Medium F12 (DMEM-F12; Biosera, Inc., Manila, Philippines), and the THP-1 cells were cultured with Roswell Park Memorial Institute (RPMI) 1640 medium with $10 \%$ fetal bovine serum, and 100 $\mathrm{U} / \mathrm{mL}$ penicillin, $100 \mu \mathrm{g} / \mathrm{mL}$ streptomycin and $1 \% 2 \mathrm{mM} \mathrm{L-glu-}$ tamate (all Biosera, Inc., Manila, Philippines) at $37^{\circ} \mathrm{C}$ and $5 \%$ $\mathrm{CO} 2$ in a cell culture incubator. The cells were checked daily and subcultured when they reached $70 \%$ to $80 \%$ confluence. Third-passage cells were used to perform the experiments.

\section{Preconditioning}

To create fully differentiated THP-1 macrophage cells, 3×105 cells $/ \mathrm{mL}$ THP-1 cells were seeded in each well of a 6-well culture plate and stimulated with $10 \mathrm{ng} / \mathrm{mL}$ phorbol 12-myristate-13-acetate (PMA) (MilliporeSigma, Burlington, MA, USA) for 24 hours. Next, 3x105 AD-MSCs were seeded in each well of another set of 6-well culture plates and cultured for 24 hours. The medium was replaced and nonadherent cells were removed. To precondition the cells, $50 \mathrm{ng} / \mathrm{mL}$ IFN- $\gamma, 40 \mathrm{ng} / \mathrm{mL}$ IL-4, and $40 \mathrm{ng} / \mathrm{mL}$ IL-10 (all Reprokine Ltd., Congers, NY, USA) were added to the appropriate wells and left to culture for 24 hours. The same number of cells were cultured as unconditioned controls. After incubation, the cells to be used for flow cytometry analysis were detached using Accutase solution (Biosera, Inc., Manila, Philippines), and the cells to be used in the quantitative polymerase chain reaction (qPCR) analysis were collected using a cell scraper. All of the experiments were performed in triplicate.

\section{Flow cytometry}

Anti-human CD86/B7-2 fluorescein isothiocyanate (FITC) (clone: BU63) and CD274/PD-L1 phycoerythrin (PE) (clone: 29E.2A3) fluorescent-labeled antibodies were purchased from EXBIO Praha, a.s., Vestec, Czech Republic. Anti-human CD80/ B7-1 PE.Cy5 (clone: 1D10), CD137L/4-1BBL PE (clone: 5F4), CD252/OX40L PE (clone: 11C3.1), CD275/ICOSL (clone: 2D3), HLA-A,B,C FITC (clone: W6/32), and HLA-DR,DP,DQ FITC (clone: Tü39) fluorescent labeled antibodies were purchased from Biolegend Inc., San Diego, CA, USA. The IFN- $\gamma$, IL-4, and IL-10 stimulated cells and the unstimulated cells were stained according to the manufacturer's protocol and analyzed using an Accuri C5 flow cytometer (BD Biosciences, San Jose, CA, USA). FlowJo v10 software (FlowJo LLC, Ashland, OR, USA) was used to perform all of the analyses.

\section{Quantitative polymerase chain reaction}

Total RNA isolation was performed in accordance with the protocol provided for the Purelink RNA MiniKit (Cat. no: 12183018A; Thermo Fisher Scientific, Inc., Waltham, MA, USA), and once the 
complementary DNA was synthesized using the high-capacity RNA-to-cDNA kit (Cat. no: 4368814; Invitrogen Corp., Carlsbad, CA, USA). Forward and reverse primers of CD80/B7-1, CD86/B72, CD137L/4-1BBL, CD252/OX40L, CD274/PD-L1, CD275/ICOSL, HLA-ABC, and HLA-DR genes were purchased from Sentegen Biotech, Ankara, Turkey (Table 1). SYBR Green PCR Master Mix (Cat. no: 4344463; ThermoFisher Scientific, Inc., Waltham, MA, USA) was used to determine change in gene expressions and the reactions were assessed using the StepOnePlus Real-Time PCR System (Cat. no: 4376600 Applied Biosystems, Foster City, CA, USA). GAPDH was used as a reference gene, and the relative gene expression differences were calculated using the delta-delta cycle threshold (CT) method. The primers of the genes used in the qPCR analysis are shown in Table 1.

\section{Statistical analysis}

Data obtained from flow cytometry and qPCR analysis were evaluated using Prism v. 7.0 software (GraphPad Software, San Diego, CA, USA). The Shapiro-Wilk test was used to ana- lyze the distribution of data. Data with a normal distribution were compared using ordinary one-way analysis of variance, and those without normal distribution were evaluated using the Kruskal-Wallis method. Results of $p<0.05$ were considered statistically significant.

\section{Results}

Microscopic evaluation was performed to observe the effects of cytokines on cell morphology. IFN- $\gamma$ and IL-10-stimulated THP-1 cells displayed spindle morphology, while the stimulated IL-4 cells and the cells that had not been stimulated and had a more round morphology. Cytokine stimuli did not lead to an observable difference in the morphology of MSCs. The alterations in the microscopic images of THP-1 and AD-MSCs following cytokine stimulation are shown in Figure 1.

\section{Flow cytometry}

Positive cell frequencies (PCFs) were evaluated using flow cytometry to observe molecule expression changes in un-

Table 1. Table of primers used in quantitative polymerase chain reaction analysis

\begin{tabular}{lll}
\hline Gene & Forward & Reverse \\
\hline CD80 (B7-1) & AGGAACACCCTCCAATCTCTG & GGTCAAAAGTGAAAGCCAACA \\
CD86 (B7-2) & CTGCTCATCTATACACGGTTACC & GGAAACGTCGTACAGTTCTGTG \\
CD137L (4-1BB) & TCAGGCTCCGTTTCACTTG & CAGGTCCACGGTCAAAGC \\
CD252 (OX40L) & TGATGACTGAGTTGTTCTGCACC & CCTACATCTGCCTGCACTTCTC \\
CD274 (PD-L1) & TATGGTGGTGCCGACTACAA & TGCTTGTCCAGATGACTTCG \\
CD275 (ICOSL) & CCCAGGACGAGCAGAAGTTT & TGAAGTTGCTGCCACATGC \\
HLA-ABC & TGGGAGCTGTCTTCCCAGCCC & CCACATCACGGCAGCGACCA \\
HLA-DR & AGACAAGTTCACCCCACCAG & AGCATCAAACTCCCAGTGCT \\
GAPDH & GCCGCATCTTCTTTTGCGTC & GACGAACATGGGGGCATCAG \\
\hline
\end{tabular}

CD: Cluster of differentiation; GAPDH: Gliseraldehid 3-Fosfat Dehidrogenaz; HLA: Human leukocyte antigen; ICOSL: Inducible T cell costimulatory ligand; PD: Programmed death ligand-1.
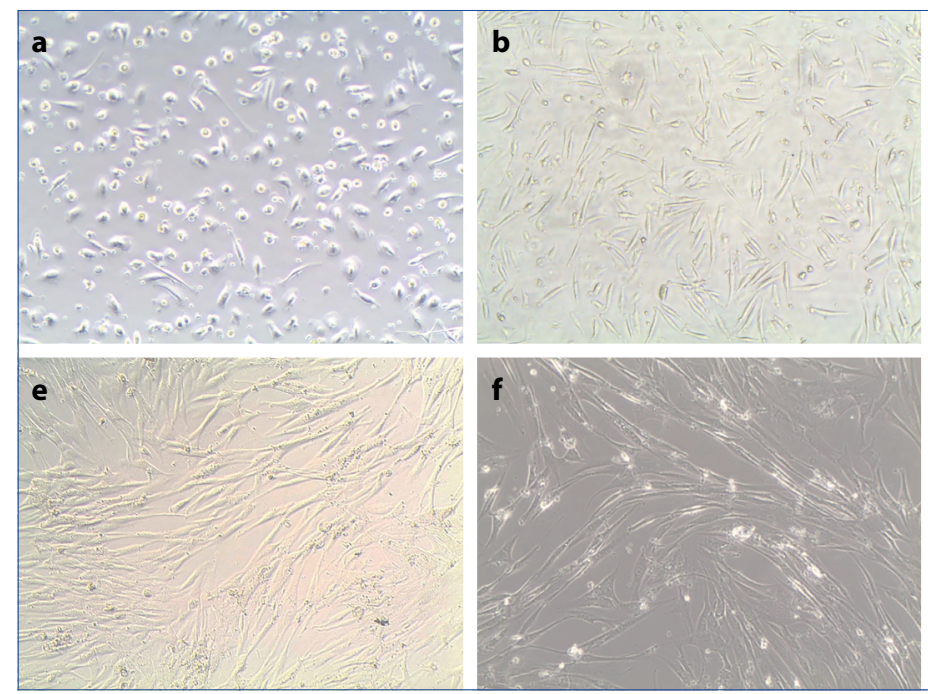
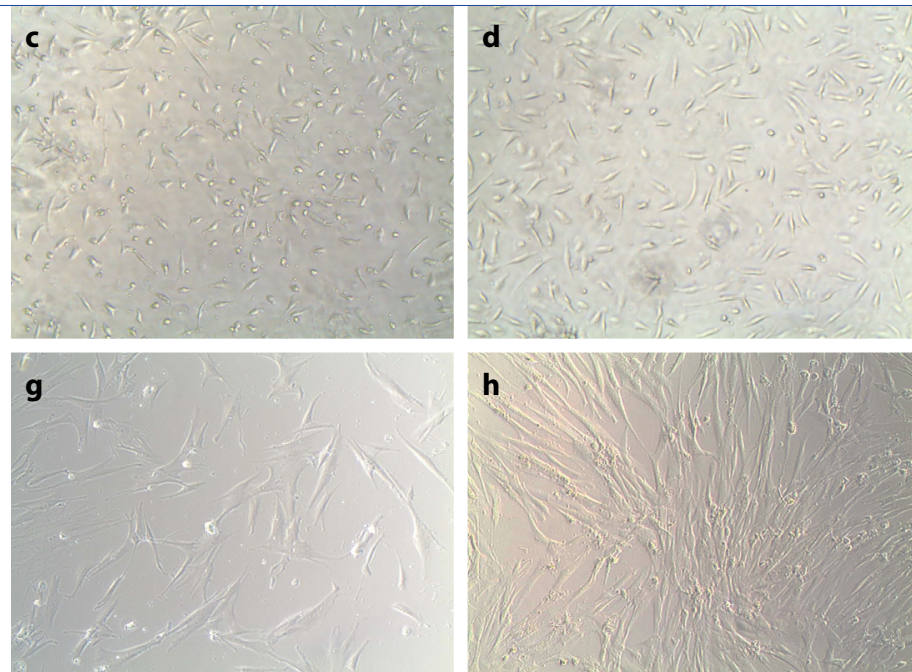

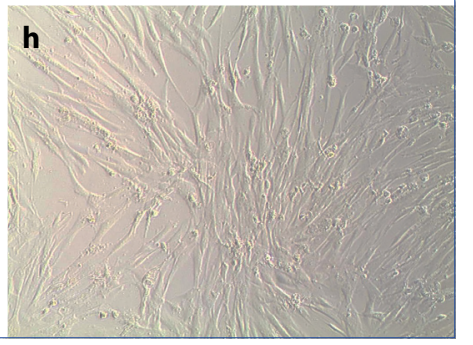

Figure 1. Microscopy images of (a) unconditioned, (b) preconditioned with interferon gamma (IFN- $\gamma$ ), (c), interleukin (IL) 4, (d) IL-10 human acute monocytic leukemia cell line (THP-1) macrophages, and (e) unconditioned, (f) preconditioned with IFN- $\gamma$, (g) IL-4, (h) and IL-10 adipose tissue mesenchymal stem cells. 
conditioned and cytokine-preconditioned cells. Histogram graphs of the flow cytometry analysis are shown in Figure 2, and group comparison charts are provided in Figure 3. The PCFs obtained from flow cytometry analysis of all groups are summarized in Table 2. It was observed that the frequencies of CD80 ( $p=0.0003), C D 86(p<0.0001), C D 137 L(p<0.0001)$, CD252 $(p=0.0003), C D 274(p=0.0077), C D 275(p<0.0001)$, and HLA-II $(p<0.0001)$ PCFs of THP-1 macrophages were significantly higher than those of the AD-MSCs, while HLA-I demonstrated no significant difference ( $p=0.1506)$ (Fig. 3).
It was also noted that cytokine stimuli applied to THP-1 macrophages created significant changes in PCFs. The frequencies of CD80, CD86, CD252, CD274, and CD275 PCFs of THP-1 macrophages significantly increased with IFN $-\gamma(p<0.0001, p=0.0181$, $p=0.0010, p<0.0001$, and $p<0.0001$ respectively), but significantly decreased with IL-10 $(p=0.0004, p<0.0001, p<0.0001$, $\mathrm{p}=0.0011$, and $\mathrm{p}<0.0001$ respectively). IL-4 stimulation significantly increased the CD86 $(p<0.0001)$ and CD274 $(p<0.0001)$ PCFs, but significantly reduced the CD80 ( $p=0.0012)$ PCFs and had no significant effect on other costimulatory molecules.

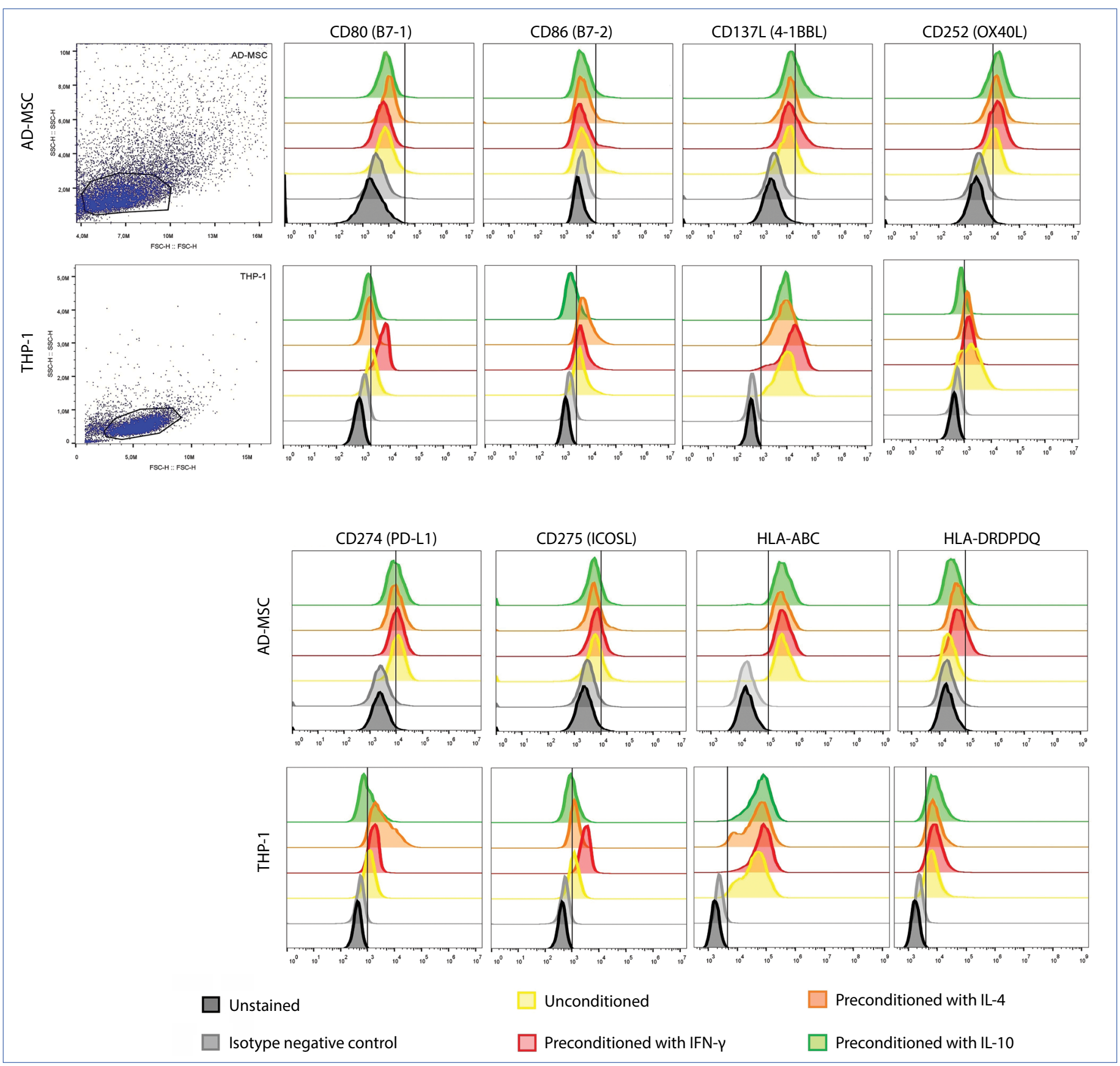

Figure 2. Histogram graphs obtained from flow-cytometry analysis of human acute monocytic leukemia cell line (THP-1) macrophages and adipose tissue mesenchymal stem cells (AD-MSCs).

HLA: Human leukocyte antigen; ICOSL: Inducible T cell costimulator ligand; IFN- $\gamma$ : Interferon gamma; IL: Interleukin; PD-L1: Programmed death-ligand. 
Table 2. Table of positive cell frequencies obtained from flow cytometry analysis of all groups

\begin{tabular}{|c|c|c|c|c|c|c|c|c|}
\hline & \multicolumn{4}{|c|}{ THP-1 macrophages } & \multicolumn{4}{|c|}{ AD-MSCs } \\
\hline & US & IFN- $\boldsymbol{\gamma}$ & IL-4 & IL-10 & US & IFN-p & IL-4 & IL-10 \\
\hline CD80 (B7-1) & $52.3 \pm 7.56$ & $92.9 \pm 0.92$ & $28.3 \pm 4.23$ & $24.6 \pm 3.70$ & $0.86 \pm 0.13$ & $0.06 \pm 0.04$ & $0.68 \pm 0.11$ & $0.21 \pm 0.05$ \\
\hline CD86 (B7-2) & $69.1 \pm 2.28$ & $79.0 \pm 4.17$ & $89.0 \pm 2.57$ & $20.5 \pm 2.96$ & $12.0 \pm 1.67$ & $5.84 \pm 0.8$ & $8.40 \pm 1.16$ & $6.1 \pm 0.84$ \\
\hline CD137L (4-1BBL) & $97.7 \pm 1.25$ & $96.2 \pm 1.20$ & $97.5 \pm 1.05$ & $97.5 \pm 1.51$ & $18.9 \pm 2.6$ & $30.5 \pm 4.2$ & $19.81 \pm 2.7$ & $29.9 \pm 3.2$ \\
\hline CD252 (OX40L) & $66.0 \pm 2.51$ & $76.9 \pm 1.76$ & $72.3 \pm 2.77$ & $17.8 \pm 0.53$ & $45.4 \pm 1.9$ & $67.6 \pm 1.7$ & $63.2 \pm 2.6$ & $68.9 \pm 2.6$ \\
\hline CD274 (PD-L1) & $61.8 \pm 3.50$ & $87.0 \pm 3.17$ & $91.9 \pm 2.92$ & $44.2 \pm 4.05$ & $51.6 \pm 0.66$ & $54.9 \pm 1.27$ & $42.35 \pm 1.63$ & $42.9 \pm 1.29$ \\
\hline CD275 (ICOSL) & $65.6 \pm 3.01$ & $95.4 \pm 0.96$ & $70.3 \pm 2.88$ & $41.8 \pm 1.35$ & $10.8 \pm 0.35$ & $21.2 \pm 0.83$ & $10.9 \pm 0.80$ & $8.47 \pm 0.64$ \\
\hline HLA-I & $95.5 \pm 1.49$ & $96.7 \pm 1.43$ & $95.1 \pm 1.67$ & $96.6 \pm 1.35$ & $93.6 \pm 1.12$ & $93.4 \pm 1.02$ & $90.9 \pm 1.18$ & $93.1 \pm 0.89$ \\
\hline HLA-II & $81.3 \pm 1.40$ & $88.0 \pm 1.48$ & $87.2 \pm 1.48$ & $86.1 \pm 1.49$ & $0.94 \pm 0.27$ & $18.2 \pm 2.27$ & $13.6 \pm 1.71$ & $2.92 \pm 0.43$ \\
\hline
\end{tabular}

The data in the table are listed as mean and SD and were obtained from 3 independent experiments. AD-MSCs: Adipose-derived mesenchymal stem cells; HLA: Human leukocyte antigen; ICOSL: Inducible T cell costimulator ligand; IFN- $\gamma$ : Interferon gamma; IL: Interleukin; PD-L1: Programmed death-ligand 1; THP-1: Human acute monocytic leukemia cell line; US: Unstimulated.

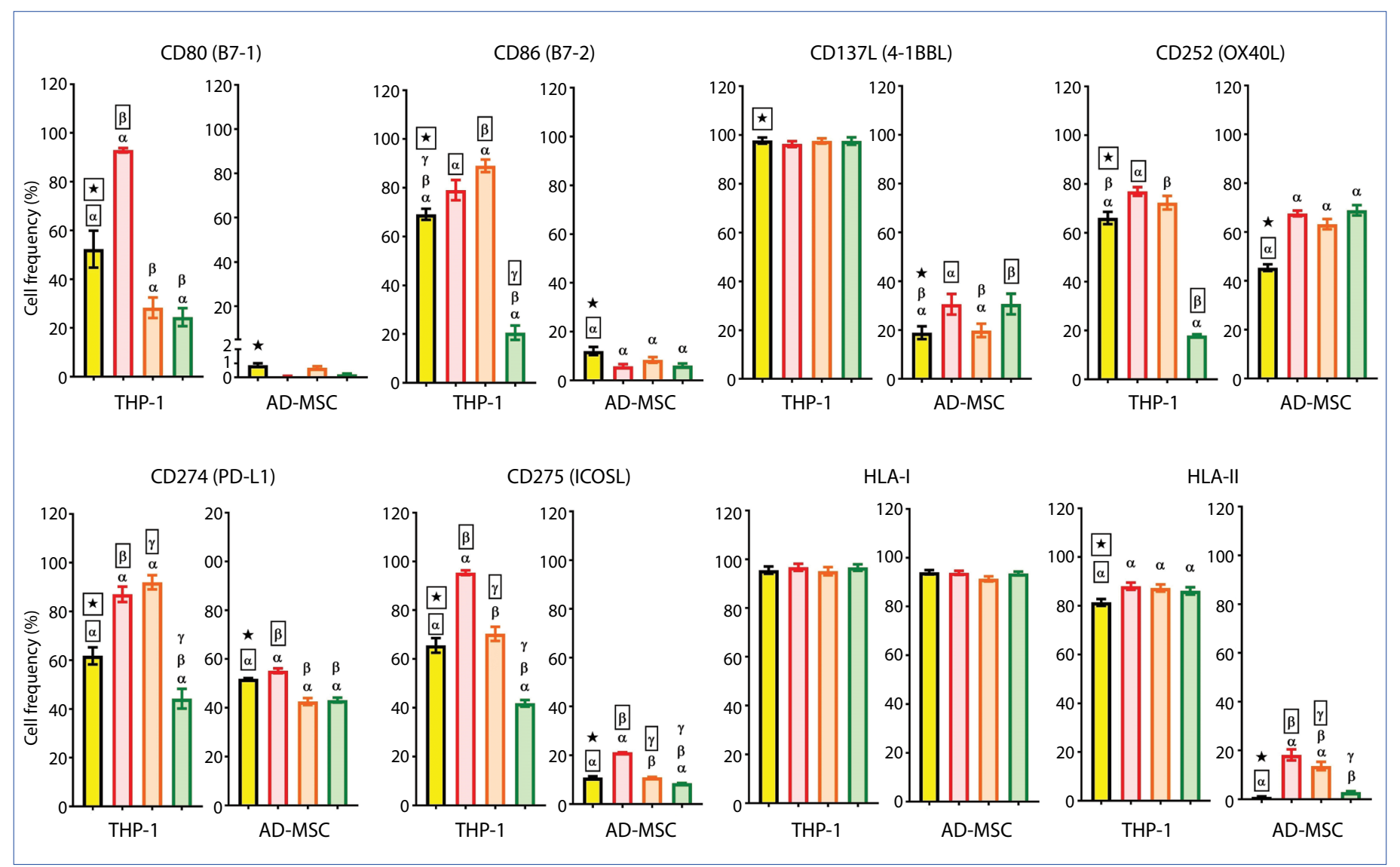

Figure 3. Comparison charts of unconditioned (yellow), preconditioned with interferon gamma (IFN- $\gamma$ ) (red), interleukin (IL) 4 (orange), and IL-10 (green)-positive cell frequencies obtained from flow cytometry analysis of all groups. The data are presented as mean and SD. There is a statistically significant difference $(p<0.05)$ between the columns marked with the same symbol shown in the box.

Compared to THP-1 macrophages, a relatively very small population of AD-MSCs was positive for CD80, CD86, CD137L, CD275 and HLA-II (Fig. 3). However, it was observed that the cytokine stimuli caused statistically significant changes in these molecule expressions. CD86 PCFs were significantly decreased by all cytokines $(p=0.0009, p=0.0212$, and $p=0.0011$ respectively), and
CD137L PCFs were significantly increased with IFN- $\gamma(p=0.0104)$ and IL-10 ( $p=0.0136)$. CD252 PCFs were significantly increased by all cytokines $(p<0.0001, p<0.0001$, and $p<0.0001$ respectively). CD274 PCFs were significantly decreased by IL-4 $(p<0.0001)$ and IL-10 $(p=0.0001)$, but increased by IFN- $\gamma(p=0.0483) . C D 275$ PCFs were significantly decreased by IL-10 $(p=0.0114)$, but in- 
Table 3. Table of positive cell frequencies obtained from flow cytometry analysis of all groups

\begin{tabular}{|c|c|c|c|c|c|c|c|c|}
\hline & \multicolumn{4}{|c|}{ THP-1 Macrophages } & \multicolumn{4}{|c|}{ AD-MSCs } \\
\hline CD80 (B7-1) & $52.3 \pm 7.56$ & $92.9 \pm 0.92$ & $28.3 \pm 4.23$ & $24.6 \pm 3.70$ & $0.86 \pm 0.13$ & $0.06 \pm 0.04$ & $0.68 \pm 0.11$ & $0.21 \pm 0.05$ \\
\hline CD86 (B7-2) & $69.1 \pm 2.28$ & $79.0 \pm 4.17$ & $89.0 \pm 2.57$ & $20.5 \pm 2.96$ & $12.0 \pm 1.67$ & $5.84 \pm 0.8$ & $8.40 \pm 1.16$ & $6.1 \pm 0.84$ \\
\hline CD137L (4-1BBL) & $97.7 \pm 1.25$ & $96.2 \pm 1.20$ & $97.5 \pm 1.05$ & $97.5 \pm 1.51$ & $18.9 \pm 2.6$ & $30.5 \pm 4.2$ & $19.81 \pm 2.7$ & $29.9 \pm 3.2$ \\
\hline CD252 (OX40L) & $66.0 \pm 2.51$ & $76.9 \pm 1.76$ & $72.3 \pm 2.77$ & $17.8 \pm 0.53$ & $45.4 \pm 1.9$ & $67.6 \pm 1.7$ & $63.2 \pm 2.6$ & $68.9 \pm 2.6$ \\
\hline CD275 (ICOSL) & $65.6 \pm 3.01$ & $95.4 \pm 0.96$ & $70.3 \pm 2.88$ & $41.8 \pm 1.35$ & $10.8 \pm 0.35$ & $21.2 \pm 0.83$ & $10.9 \pm 0.80$ & $8.47 \pm 0.64$ \\
\hline HLA-I & $95.5 \pm 1.49$ & $96.7 \pm 1.43$ & $95.1 \pm 1.67$ & $96.6 \pm 1.35$ & $93.6 \pm 1.12$ & $93.4 \pm 1.02$ & $90.9 \pm 1.18$ & $93.1 \pm 0.89$ \\
\hline HLA-II & $81.3 \pm 1.40$ & $88.0 \pm 1.48$ & $87.2 \pm 1.48$ & $86.1 \pm 1.49$ & $0.94 \pm 0.27$ & $18.2 \pm 2.27$ & $13.6 \pm 1.71$ & $2.92 \pm 0.43$ \\
\hline
\end{tabular}

The data in the table are listed as mean and SD and were obtained from 3 independent experiments. AD-MSCs: Adipose-derived mesenchymal stem cells; HLA: Human leukocyte antigen; ICOSL: Inducible T cell costimulator ligand; IFN-Y: Interferon gamma; IL: Interleukin; PD-L1: Programmed death-ligand 1; THP-1: Human acute monocytic leukemia cell line; US: Unstimulated.

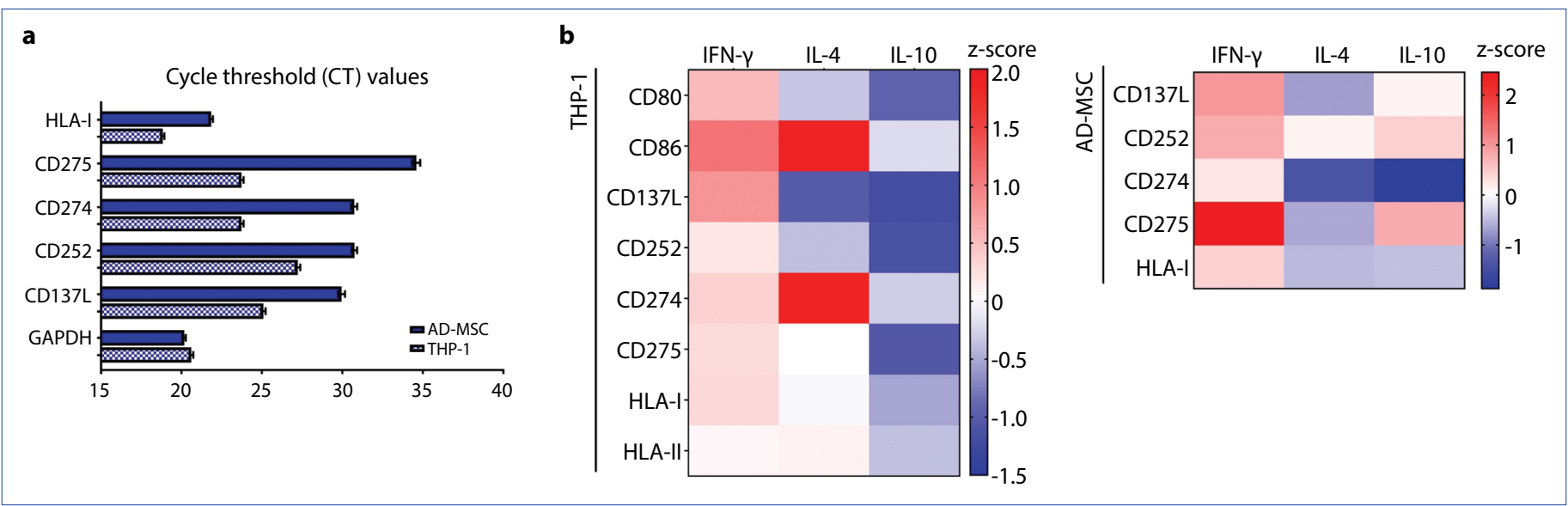

Figure 4. Comparison graphs of data from quantitative polymerase chain reaction analysis of all groups. (a) Comparison of cycle threshold data of gene expressions that can be measured in both human acute monocytic leukemia cell line (THP-1) macrophages and adipose tissue mesenchymal stem cells (AD-MSCs). (b) Heat-map graphs of the changes of the genes expressed in THP-1 macrophage and AD-MSCs with interferon gamma (IFN- $\gamma$ ), interleukin (IL) 4, and IL-10 stimuli. (delta-delta cycle threshold values were presented by converting to z-score.)

HLA: Human leukocyte antigen; IFN- $\gamma$ : Interferon gamma; IL: Interleukin.

creased by IFN- $\gamma(p<0.0001)$. HLA-II PCFs were significantly increased by only IFN- $\gamma(p<0.0001)$ and IL-4 $(p<0.0001)$.

\section{Gene expressions}

Since the CT values of CD80, CD86, and HLA-II genes of ADMSCs are $>35$, these molecules were excluded from evaluation. The GAPDH CT values of unstimulated THP-1 and ADMSCs were similar, but the CT values of MSCs were significantly higher for other molecules (Fig. 4a). This finding suggested that the gene expressions of THP-1 cells were significantly higher than those of MSCs for the molecules evaluated. When the changes caused by cytokines were evaluated, it was observed that IFN- $\gamma$ significantly increased all gene expressions in the THP-1 cells, an in contrast, IL-10 significantly reduced all gene expressions. CD86 and CD274 expressions of THP- 1 cells were significantly increased by IL-4, but expressions of other molecules were decreased significantly (Fig. 4b). Similarly,
IFN- $\gamma$ significantly increased overall gene expression of ADMSCs. IL-4 significantly reduced the expression of genes other than CD252. IL-10 significantly reduced CD274 and HLA-I expressions, but increased CD137L, CD252, and CD275 (Fig. 4b). The delta-delta CT values of all of the groups are summarized in Table 3. The $p$ values of the statistical comparisons are presented in the Table 4.

\section{Discussion}

This study was an investigation of the expression of costimulatory molecules, which have critical roles of the activation of T cells in AD-MSCs. We used macrophages differentiated from THP-1 cells as the reference APC model. It was found that the CD80, CD86, CD137L, CD252, CD274, CD275, and HLA-II PCFs of AD-MSCs were significantly lower than those of THP-1 macrophages; however, HLA-I revealed no significant difference. We found that preconditioning with IFN- $\gamma$ led to a significant 
Table 4. One-way analysis of variance with Tukey's multiple comparison test results of the delta-delta cell threshold values of all groups

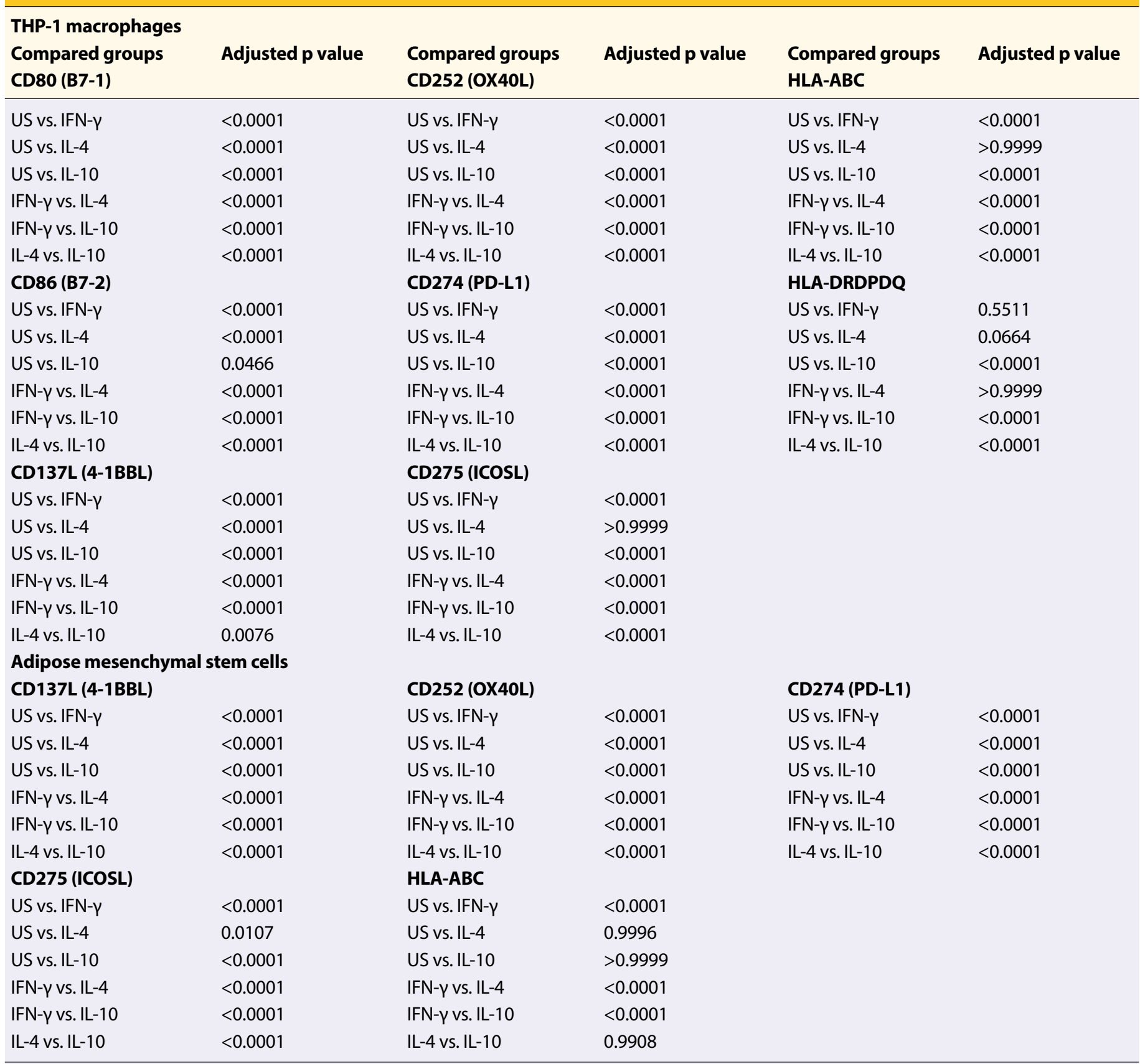

HLA: Human leukocyte antigen; ICOSL: Inducible T cell costimulator ligand; IFN- $\gamma$ : Interferon gamma; IL: Interleukin; PD-L1: Programmed death-ligand 1THP-1: Human acute monocytic leukemia cell line; US: Unstimulated.

increase in all of the molecule expressions evaluated in THP-1 macrophages, but IL-10 led to a significant decrease in the opposite direction. IL-4 caused a significant decrease in the CD80 PCFs of THP-1 cells, and a significant increase in the CD86 and CD274 PCFs. In addition, we observed that the CD80, CD86, and HLA-II PCFs of AD-MSCs were extremely when low compared with THP-1 cells. We found that IFN- $\gamma$ led to a significant increase in CD137L, CD252, CD274, and CD275 mRNA and PCFs in AD-MSCs, similar to THP-1 cells.
Three basic signals have been identified in the activation of T cells by antigen presentation. The first signal (Signal 1) for antigen-specific activation of $\mathrm{T}$ cells is provided by the interaction of the T cell receptor and peptide-HLA complexes. Antigen presentation via HLA-I is restricted to CD8 T cells, and HLA-II is restricted to CD4 T cells. Therefore, while HLA-I is expressed in all cells, HLA-Il is expressed by professional antigen presenting cells (APCs) [20]. We observed that almost all of the THP-1 macrophages and AD-MSCs were positive for HLA-I. 
Unlike THP-1 macrophages, we found that AD-MSCs did not express HLA-II; however, we detected a small but significant increase with IFN- $\gamma$ and IL-4 stimulation. This finding indicated that AD-MSCs may not present antigens to CD4 T cells.

Signal 1 alone is not sufficient for $T$ cell activation and lineage commitment. A second signal (Signal 2) provided by costimulatory molecules is necessary for complete activation [20]. CD80/B7-1 and CD86/B7-2 are costimulatory molecules that are highly expressed on macrophages and mature monocyte-derived DCs (mo-DCs) and interact with the CD28 molecule on the $T$ cell surface, enabling $T$ cell activation [21]. It has been reported that IFN- $\gamma$ significantly increases both CD80 and CD86 expressions, IL-4 leads to a more pronounced increase for CD86, but IL-10 significantly reduces expression of both molecules [22]. Our results were consistent with the literature. CD80 and CD86 expressions of THP-1 macrophages were increased significantly by IFN- $\gamma$ but were significantly decreased by IL-10. However, IL-4 significantly suppressed CD80 expression while significantly increasing CD86 expression. A limited number of studies of MSCs have shown that these cells do not express CD80 and CD86, and IFN- $\gamma$ stimulation does not affect this condition [23]. Flow cytometry analysis indicated that AD-MSCs did not express CD80, and CD86 expressions were extremely low. QPCR analysis did not reveal expression of either molecule (Fig. 3 and 4).

CD137L/4-1BBL is a costimulatory molecule commonly expressed by APCs and interacts with CD137/4-1BB found in $T$ and natural killer (NK) cells. The interaction of CD137/ CD137L not only activates T and NK cells, but APCs are differentiated in the pro-inflammatory direction [24]. A review of the current literature did not disclose another study investigating the effects of different cytokine stimuli on CD137L expression in macrophages or mo-DCs. We found that more than $90 \%$ of THP-1 macrophages were positive for CD137L, and that CD137L mean fluorescent intensity (MFI) values and mRNA expressions only increased significantly with IFN- $\gamma$ (Fig. 3 and 4). Only 1 study has demonstrated that MSCs can express CD137L and suppress T cell proliferation [25]. We found that AD-MSCs had a significantly lower but basal CD137L expression compared with THP-1 macrophages, and this expression was increased significantly by IFN- $\gamma$ and IL-10 stimuli (Fig. 3 and 4).

CD252/OX40L interacts with the CD134/OX40 located on the $T$ cell surface and produces a signal that increases the proliferation and survival of effector T cells. OX40 / OX40L interaction creates a bidirectional signal that activates both $T$ cells and APCs, and activation of APCs allows them to express more OX40L [26]. There are only a few studies that have examined the OX40L expression of both APCs and MSCs and how they change with cytokine stimulation. It has been reported that OX40L expression of microglia cells increases with IFN- $\gamma$ stimulation [27]. It has also been observed that MSCs increase regulatory $T$ cell (Treg) ratios more effectively by overexpressing OX40L [28]. We found that the OX40L expression of THP-1 macrophages increased significantly with IFN- $\gamma$ and IL-4 stimuli, but decreased significantly with IL-10. We identified OX40L expression in more than half of the AD-MSCs, and observed that expression was significantly increased by all three cytokines (Fig. 3 and 4).

CD274/PD-L1 interacts with CD279/PD-1 on the T cell surface, causing an increase in apoptosis and a decrease in activation and proliferation. This provides for the development of central and peripheral tolerance. Therefore, the PD-1/PD-L1 signal plays an important role in the pathogenesis of a wide range of diseases, such as chronic infection, autoimmune diseases, and cancer [29]. We found that the PD-L1 expression of THP1 macrophages was increased significantly by IFN- $\gamma$ and IL-4, but decreased significantly by IL- 10 . We observed that almost half of the AD-MSCs expressed PD-L1, and that this expression increased significantly with IFN- $\gamma$, but decreased significantly with IL-4 and IL-10.

CD275/ICOSL is the ligand of the CD278/ICOS molecule found in T cells and provides the effector and memory cells to proliferate and survive. It plays a critical role in antibody production, particularly by regulating follicular T cells [30]. ICOSL expression of DCs has been shown to increase significantly with IFN- $\gamma$ and IL-4 [31]. It has been demonstrated that MSCs are able to express ICOSL and that the Treg induction capacity is proportional to the expressed ICOSL ratio [32]. We found that the ICOSL expression of THP-1 macrophages increased signifcantly with IFN- $\gamma$ but decreased with IL-10. However, we also observed that in MSCs, ICOSL expression was limited to a small population, and this expression was significantly increased by IFN- - , but decreased by IL-10.

The third and final basic signal in $\mathrm{T}$ cell activation is created by cytokines found in the environment, such as IFN- $\gamma$, IL-4, IL10 , or TGF- $\beta$ [33]. MSCs could provide effective immune suppression through the powerful molecules they secrete; that is, MSCs are strong Signal 3- producer cells [2,3]. However, this effect is unfortunately limited by the presence of MSCs. Tolerogenic DCs produce suppressive molecules like MSCs, but unlike the MSCs, they play a critical role in the formation of peripheral tolerance because they express HLA-II [19]. MSCs have provided promising improvements in clinical trials examining immune pathologies such as graft-versus-host disease, systemic lupus erythematosus, and multiple sclerosis, which can be fatal and for which current treatment approaches are inadequate [34]. However, the results of these trials have shown that the effects of MSCs were temporary [35]. Therefore, it may be that the immunomodulation effects of MSCs are insufficient to establish an antigen-specific tolerance. Although the preconditioning approach improves the immunomodulation properties of MSCs, our findings indicate that this approach did not significantly change the costimulatory molecule expressions. This suggests that, since Signal 1 and Signal 2 were not properly generated by MSCs, they may be insufficient to formulate tolerance. HLA-I expression of MSCs could enable them to interact with CD8 T cells, but the absence of 
costimulatory molecules will cause CD8 T cells to be anergic. MSCs need expression of HLA-II for anergy-inducing potential to occur on CD4 T cells. The transfer of HLA-II molecules to MSCs through gene engineering could enable these cells to form a broader and stronger tolerance, including CD4 T cells.

\section{Conclusion}

In conclusion, our data indicated that although the MSCs are potent immunomodulatory cells, the antigen presentation capabilities were not comparable to those of professional antigen presenting cells. In addition, the costimulatory molecule expressions of MSCs may not be significantly altered with a preconditioning approach. However, the transfer of costimulatory molecules through gene engineering could enable MSCs to develop a more effective and lasting tolerance potential.

Acknowledgments: We would like to thank Dr. Ayse Nalbantsoy from Ege University, Faculty of Engineering, Bioengineering Department for her help with the flow cytometry analysis.

Conflict of Interest: The authors declared no potential conflicts of interest with respect to the research, authorship, and/or publication of this article.

Financial Disclosure: The Scientific Research Projects Commission of Manisa Celal Bayar University provided financial support for the project, reference number 2019/087.

Peer-review: Externally peer-reviewed.

Authorship Contributions: Concept - A.T.O., R.B.O.O., C.K.; Design - A.T.O., M.O., B.C.; Supervision - A.T.O., K.O., C.K.; Funding - C.K.; Materials - A.T.O., M.O., B.C.; Data collection \&/or processing - A.T.O., M.O., B.C., E.D.; Analysis and/or interpretation - A.T.O., M.O., R.B.O.O., B.C.; Literature search - A.T.O., M.O., R.B.O.O.; Writing - A.T.O., M.O., R.B.O.O., B.C., K.O., E.D., C.K.; Critical review A.T.O., M.O., R.B.O.O., C.K.

\section{References}

1. Özdemir AT, Özgül Özdemir RB, Kırmaz C, Sarıboyacı AE, Ünal Halbutoğlları ZS, Özel C, et al. The paracrine immunomodulatory interactions between the human dental pulp derived mesenchymal stem cells and CD4 T cell subsets. Cell Immunol 2016;310:108-15. [CrossRef]

2. Özdemir RBÖ, Özdemir AT, Sarıboyacı AE, Uysal O, Tuğlu Mi, Kırmaz C. The investigation of immunomodulatory effects of adipose tissue mesenchymal stem cell educated macrophages on the CD4 T cells. Immunobiology 2019;224(4):585-94. [CrossRef]

3. Weiss ARR, Dahlke MH. Immunomodulation by mesenchymal stem cells (MSCs): mechanisms of action of living, apoptotic, and Dead MSCs. Front Immunol 2019;10:1191. [CrossRef]

4. Corsello T, Amico G, Corrao S, Anzalone R, Timoneri F, Lo lacono $\mathrm{M}$, et al. Wharton's jelly mesenchymal stromal cells from human umbilical cord: a close-up on immunomodulatory molecules featured in situ and in vitro. Stem Cell Rev Rep 2019;15(6):900-18. [CrossRef]

5. Davies LC, Heldring N, Kadri N, Le Blanc K. Mesenchymal stromal cell secretion of programmed death-1 ligands regulates $T$ cell mediated immunosuppression. Stem Cells 2017;35(3):766-76. [CrossRef]

6. Özdemir RBÖ, Özdemir AT, Kirmaz C, Tuğlu Mi, Şenol Ö, Özverel $C S$, et al. The effects of mesenchymal stem cells on the IDO, HLA-G and PD-L1 expression of breast tumor cells MDAMB-231 and MCF-7. Arch Clin Exp Med 2019;4(3):132-7.

7. Dazzi F, Krampera M. Mesenchymal stem cells and autoimmune diseases. Best Pract Res Clin Haematol 2011;24(1):4957. [CrossRef]

8. Hu C, Li L. Preconditioning influences mesenchymal stem cell properties in vitro and in vivo. J Cell Mol Med 2018;22(3):142842. [CrossRef]

9. Seo Y, Shin TH, Kim HS. Current strategies to enhance adipose stem cell function: an update. Int J Mol Sci 2019;20(15):3827.

10. Guan Q, Li Y, Shpiruk T, Bhagwat S, Wall DA. Inducible indoleamine 2,3-dioxygenase 1 and programmed death ligand 1 expression as the potency marker for mesenchymal stromal cells. Cytotherapy 2018;20(5):639-49. [CrossRef]

11. Liu KJ, Wang CJ, Chang CJ, Hu HI, Hsu PJ, Wu YC, et al. Surface expression of HLA-G is involved in mediating immunomodulatory effects of placenta-derived multipotent cells (PDMCs) towards natural killer lymphocytes. Cell Transplant 2011;20(1112):1721-30. [CrossRef]

12. Crop MJ, Baan CC, Korevaar SS, ljzermans JN, Pescatori M, Stubbs AP, et al. Inflammatory conditions affect gene expression and function of human adipose tissue-derived mesenchymal stem cells. Clin Exp Immunol 2010;162(3):474-86. [CrossRef]

13. Han X, Yang Q, Lin L, Xu C, Zheng C, Chen X, et al. Interleukin-17 enhances immunosuppression by mesenchymal stem cells. Cell Death Differ $2014 ; 21(11): 1758-68$. [CrossRef]

14. Prasanna SJ, Gopalakrishnan D, Shankar SR, Vasandan AB. Pro-inflammatory cytokines, IFNgamma and TNFalpha, influence immune properties of human bone marrow and Wharton jelly mesenchymal stem cells differentially. PLoS One 2010;5(2):e9016. [CrossRef]

15. Schorer M, Kuchroo VK, Joller N. Role of co-stimulatory molecules in T Helper Cell differentiation. Adv Exp Med Biol 2019;1189:153-77. [CrossRef]

16. Batten P, Sarathchandra P, Antoniw JW, Tay SS, Lowdell MW, Taylor PM, et al. Human mesenchymal stem cells induce T cell anergy and downregulate $\mathrm{T}$ cell allo-responses via the $\mathrm{TH} 2$ pathway: relevance to tissue engineering human heart valves. Tissue Eng 2006;12(8):2263-73. [CrossRef]

17. van Megen KM, van 't Wout ET, Lages Motta J, Dekker B, Nikolic $\mathrm{T}$, Roep BO. Activated mesenchymal stromal cells process and present antigens regulating adaptive immunity. Front Immunol 2019;10:694. [CrossRef]

18. Chen L, Flies DB. Molecular mechanisms of T cell co-stimulation and co-inhibition. Nat Rev Immunol 2013;13(4):227-42.

19. Hubo M, Trinschek B, Kryczanowsky F, Tuettenberg A, Steinbrink $\mathrm{K}$, Jonuleit $\mathrm{H}$. Costimulatory molecules on immunogenic 
versus tolerogenic human dendritic cells. Front Immunol 2013;4:82. [CrossRef]

20. Kotsias F, Cebrian I, Alloatti A. Antigen processing and presentation. Int Rev Cell Mol Biol 2019;348:69-121. [CrossRef]

21. Van Gool SW, Vandenberghe P, de Boer M, Ceuppens JL. CD80, CD86 and CD40 provide accessory signals in a multiple-step Tcell activation model. Immunol Rev 1996;153:47-83. [CrossRef]

22. Ambarus CA, Krausz S, van Eijk M, Hamann J, Radstake TR, Reedquist KA, et al. Systematic validation of specific phenotypic markers for in vitro polarized human macrophages. J Immunol Methods 2012;375(1-2):196-206. [CrossRef]

23. Klyushnenkova E, Mosca JD, Zernetkina V, Majumdar MK, Beggs KJ, Simonetti DW, et al. T cell responses to allogeneic human mesenchymal stem cells: immunogenicity, tolerance, and suppression. J Biomed Sci 2005;12(1):47-57. [CrossRef]

24. Dharmadhikari B, Wu M, Abdullah NS, Rajendran S, Ishak ND, Nickles E, et al. CD137 and CD137L signals are main drivers of type 1, cell-mediated immune responses. Oncoimmunology 2015;5(4):e1113367. [CrossRef]

25. Taechangam N, lyer SS, Walker NJ, Arzi B, Borjesson DL. Mechanisms utilized by feline adipose-derived mesenchymal stem cells to inhibit T lymphocyte proliferation. Stem Cell Res Ther 2019;10(1):188. [CrossRef]

26. Webb GJ, Hirschfield GM, Lane PJ. OX40, OX40L and autoimmunity: a comprehensive review. Clin Rev Allergy Immunol 2016;50(3):312-32. [CrossRef]

27. Wang Y, Li M, Song M, Xu X, Xiong J, Yang X, et al. Expression of OX40 ligand in microglia activated by IFN-gamma sus- tains a protective CD4+ T-cell response in vitro. Cell Immunol 2008;251(2):86-92. [CrossRef]

28. Liu T, Zhang Y, Shen Z, Zou X, Chen X, Chen L, et al. Immunomodulatory effects of OX40Ig gene-modified adipose tissue-derived mesenchymal stem cells on rat kidney transplantation. Int J Mol Med 2017;39(1):144-52. [CrossRef]

29. Kythreotou A, Siddique A, Mauri FA, Bower M, Pinato DJ. PDL1. J Clin Pathol 2018;71(3):189-94. [CrossRef]

30. Wikenheiser DJ, Stumhofer JS. ICOS co-stimulation: friend or foe? Front Immunol 2016;7:304. [CrossRef]

31. Aicher A, Hayden-Ledbetter M, Brady WA, Pezzutto A, Richter $G$, Magaletti $D$, et al. Characterization of human inducible costimulator ligand expression and function. J Immunol 2000;164(9):4689-96. [CrossRef]

32. Lee HJ, Kim SN, Jeon MS, Yi T, Song SU. ICOSL expression in human bone marrow-derived mesenchymal stem cells promotes induction of regulatory T cells. Sci Rep 2017;7:44486.

33. Zhu J, Yamane H, Paul WE. Differentiation of effector CD4T cell populations $\left(^{*}\right)$. Annu Rev Immunol 2010;28:445-89. [CrossRef]

34. Haque N, Ramasamy TS, Kasim NHA. Mechanisms of mesenchymal stem cells for autoimmune disease treatment. In: Pham PV, editor. Stem cell transplantation for autoimmune diseases and inflammation. New York: Springer International Publishing; 2019. p. 2744. [CrossRef]

35. Lukomska B, Stanaszek L, Zuba-Surma E, Legosz P, Sarzynska S, Drela K. Challenges and controversies in human mesenchymal stem cell therapy. Stem Cells Int 2019;2019:9628536. [CrossRef] 